\title{
THE CNOC2 FIELD GALAXY REDSHIFT SURVEY
}

\author{
H.K.C. YEE, M.J. SAWICKI, R.G. CARLBERG, H. LIN \\ University of Toronto \\ Dept. of Astronomy, Toronto ON M5S 3H8, Canada \\ S.L. MORRIS \\ DAO/HIA, 5071 Saanich Rd, Victoria BC V8X $4 M 6$ Canada \\ D.R. PATTON, G.D. WIRTH \\ University of Victoria \\ Dept. of Physics \& Astronomy, Victoria BC V8W 3P6, Canada \\ C.W. SHEPHERD \\ University of Toronto \\ Dept. of Astronomy, Toronto ON M5S 3H8, Canada \\ E. ELLINGSON \\ University of Colorado \\ CASA, CB 389, Boulder CO 80309, USA \\ AND \\ D. SCHADE, R. MARZKE \\ DAO/HIA 5071 Saanich Rd, Victoria $B C$ V8X $4 M 6$ Canada
}

\section{Introduction}

Fundamental to our understanding of the universe is the evolution of structures, from galaxies to clusters of galaxies to large-scale sheets and filaments of galaxies and voids. The investigation of the evolution of large-scale structure not only provides us with the key test of theories of structure formation, but also allows us to measure fundamental cosmological parameters. The CNOC2 (Canadian Network for Observational Cosmology) Field Galaxy Redshift Survey is the first large redshift survey of faint galaxies carried out with the explicit goal of investigating the evolution of large scale structure. This survey also provides the largest redshift and photometric data set currently available for the study of galaxy population and evolution at the moderate redshift range between 0.1 and 0.6 . In this paper we describe the scope and technique of the survey, its status, and some preliminary results.

\section{Observational Strategy}

The CNOC2 survey is being conducted using the MOS arm of the MOS/SIS spectrograph at the Canada-France-Hawaii Telescope. The primary goal of the survey is to obtain a large enough sample of galaxies with high quality spectroscopic and photometric data for the purpose of studying the large scale structure at the redshift of $\sim 0.35$. We need to obtain a sample of galaxies comparable to the large nearby redshift surveys (e.g., Geller \& Huchra 1989; Shectman et al. 1996). This requires obtaining redshifts for the order of $10^{4}$ galaxies covering an area of sky subtending well over $10 \mathrm{~h}^{-1}$ $\mathrm{Mpc}$ with velocity measurements accurate to better than $100 \mathrm{~km} \mathrm{~s}^{-1}$.

To avoid being dominated by a small number of large structures, the survey covers four widely separated regions, called patches, on the sky. The patches are distributed so that two regions can be observed at any time of the year. Each patch is a mosaic of 20 MOS fields, covering $\sim 1400$ sq arcmin. The total area of the survey is $\sim 1.5 \mathrm{sq}$ degrees. Each spectroscopically defined MOS field is $\sim 9^{\prime} \times 8^{\prime}$, with about $15^{\prime \prime}$ overlapping area with the adjacent fields. The overlapping of the 


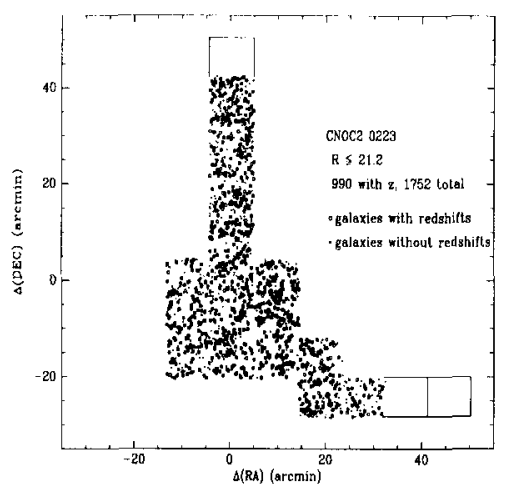

Figure 1. Field layout in a patch. Currently, 17 of 20 fields in $0223+00$ have been observed, with a total of 1310 redshifts. Symbols denote galaxies in a $R \leq 21.2$ sample.

fields minimizes the systematic uncertainties in photometric calibrations from field to field. The field layout, in a L shape with a central block, is shown in Figure 1. The maximum dimensions of the patches span $\sim 80^{\prime} \mathrm{NS}$, and $63^{\prime} \mathrm{EW}$. The patches are chosen to avoid bright $(<12 \mathrm{mag}$ ) stars, low-redshift (e.g., Abell) clusters, and other known low redshift bright objects. They have galactic latitudes between $45^{\circ}$ and $60^{\circ}$, chosen to avoid excessive Galactic extinction $(<0.05 \mathrm{mag}$ in $A_{B}$ ), yet close enough to the galactic plane to provide sufficient number of stars to overcome the star-galaxy classification problem for the MOS images which have variable point-spread-function across the field.

We utilize the observational technique developed for the CNOC1 Cluster Redshift Survey (Yee, Ellingson, \& Carlberg 1996; hereafter, YEC). Here, we describe briefly the procedure and some improvements. For the first 3 runs, two different CCDs with $15 \mu /$ pixel were used. However, for the remaining 4 runs, from which most of the data were (or will be) obtained, a STIS CCD, which has higher quantum efficiency (QE) and superior blue response, but with a larger $21 \mu$ pixel size, was used. Here, for brevity, we describe primarily the observational parameters for runs using the higher QE STIS CCD.

Images in $I, R$, Gunn $g, B$, and $U$ for each field are obtained using the imaging mode of MOS with integration times of 6 to $15 \mathrm{~min}$, obtaining average $5 \sigma$ detection magnitudes for $R$ and $B$ of 24.0 and 24.6, respectively. The $B$ and $U$ images for all the fields are taken with the blue sensitive CCD. The photometry is reduced and multicolor catalogs produced in real time at the telescope. Using the catalogs, multislit masks are designed using a computer program which allows one to prioritize the sample in various ways and optimize the number of slit placements. The slits have a width of $1{ }^{\prime \prime} 3$ and a minimum length of $11^{\prime \prime}$. The masks are cut using LAMA, a computer controlled laser cutting machine, and are available for the spectroscopic observation within as little as 3 hours after the direct imaging observation. The primary spectroscopic sample is the union set of galaxies with $R<21.5$ or $B<22.5$. This allows us to construct unbiased $R$ and $B$ samples from the redshift catalogs.

The spectroscopy observations are done using the B300 grism providing a resolution of $\sim 15 \AA$. A band limiting filter is used to shorten the spectrum so that more slits can be placed on each mask, with typical numbers ranging from 85 to 110 . The wavelength coverage of the filter is $4300 \AA$ to $6300 \AA$, allowing for the unbiased coverage of important spectroscopic features within the target redshift range of $0.10<z<0.55$ for the survey.

Two masks are used for each field. Mask A covers primarily galaxies brighter than $R=20.0$, with a total integration time of $40 \mathrm{~min}$. For mask B, which has an integration time of $80 \mathrm{~min}$, the highest priorities are assigned to galaxies with $R$ between 20 and 21.5. Fainter objects are designed into the masks whenever possible. The masks are designed so that about $25 \%$ of the galaxies observed spectroscopically are common to both masks. These redundant observations serve two purposes: first, for the fainter objects, the summed spectra provide improved signal-to-noise ratio; second, objects with redshifts determined independently from both masks provide a rigorous empirical 


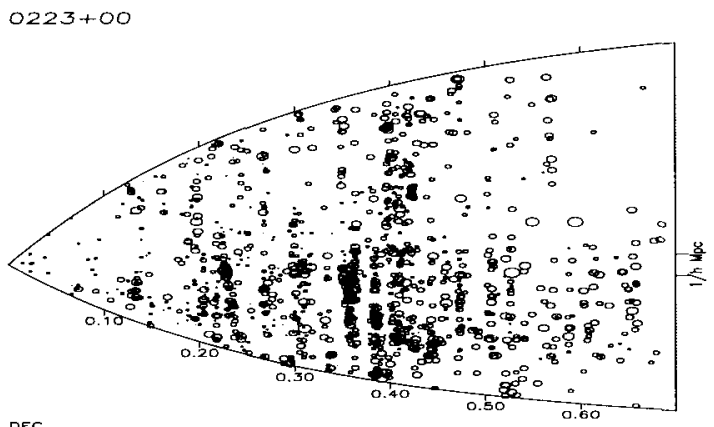

Figure 2. Wedge Diagram for the patch $0223+00$. The tick marks on the vertical axis denote $1 h^{-1} \mathrm{Mpc}$, while the horizontal axis is marked by redshift. Note the sheet-like structures spanning across the whole field in the Dec axis. The uneveness of the density of points across the structures is due to an unequal numbers of fields projected

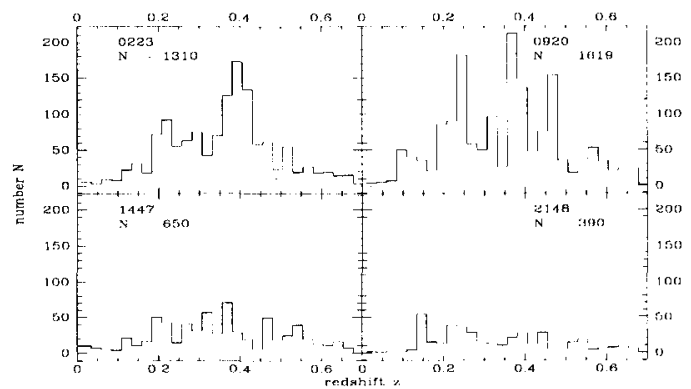

Figure 3. Histograms of the $z$ distributions from the 3969 redshifts currently available in the 4 patches

check for the velocity accuracy and the probability of catastrophic redshift errors. The two-mask strategy also allows us to compensate for the under-sampling of close pairs that occurs' when only one mask is used.

The total time required, using the more efficient $\mathrm{CCD}$ and including overhead, for the 5 colors and 2 spectroscopic masks per MOS field is about $3.5 \mathrm{hrs}$, yielding typically 80 to 90 redshifts. Thus, the complete survey of 80 fields requires about 27 clear nights, providing about 6500 redshifts with a mean $z$ of 0.35 . At the intended survey spectroscopic limit of $R=21.5$, the cumulative sampling rate for the redshift sample is about 0.50 of the photometric sample, while the differential completeness is about 0.25 .

\section{Catalog Creation}

The data reduction procedure follows basically that outlined in YEC for the CNOC1 survey. Photometry is performed using an improved version of PPP (Yee 1991). Velocities are determined using cross-correlation. With 5 photometric colors we are also able to verify the redshift assignment.

Because the survey uses a sparse sampling strategy, accurate weighting factors for each galaxy are essential for proper interpretation. Although this has been discussed in detail in YEC where magnitude, geometric, and color weights were presented, additional effort has been put into better defining the selection function due to the limited redshift range over which the survey is sensitive. Photometric redshifts will be used to estimate the survey completeness as a function of redshift, and simulations of spectra will be used to investigate how the redshift identification success rate depends on variables such as spectral signal-to-noise ratio, galaxy type, redshift, and surface brightness. The objects from all the fields in each patch are merged into a single list after correcting for distortion in the MOS image, creating a final catalog which includes photometry, redshift, and selection weights for all objects brighter than $R=24$. 


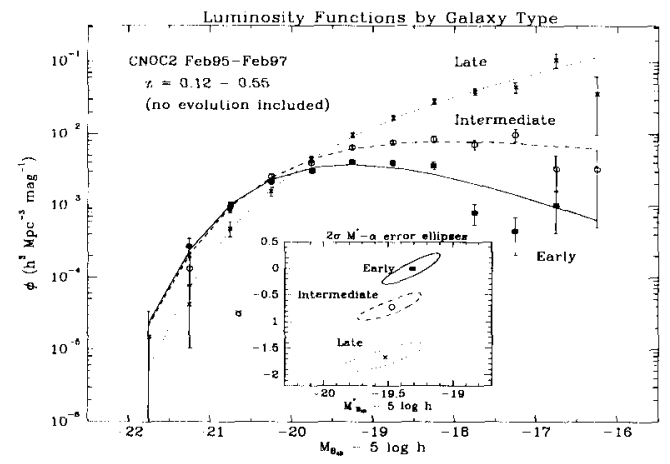

Figure 4. Preliminary luminosity function of galaxies of different population types as chosen by $B-R$ colors.

\section{Survey Status}

The survey is presently (09/97) $80 \%$ complete, and will be completed by the spring of 1998 . The current reduced sample contains $\sim 4000$ redshifts, with an additional $\sim 1000$ redshifts to be obtained from the data currently being reduced. From the redundant observations the typical rms uncertainty of the velocity determination is about $75 \mathrm{~km} \mathrm{~s}^{-1}$ in the rest frame.

Figure 1 illustrates the sky distribution of galaxies for the redshift sample in the 17 fields currently available in the $0223+00$ patch. Figure 2 shows the wedge diagram of the $0223+00$ patch. Note the many sheet-like structures in redshift space spanning the whole NS width of the patch of $\sim 10-15 h^{-1} \mathrm{Mpc}$. The redshift distributions of the current data set in the 4 patches are shown as histograms in Figure 3.

Figure 4 shows some preliminary LFs obtained from a subsample of 2768 galaxies with $R<21.2$. The galaxies are divided into early, intermediate, and late types based on $B-R$ colors. Note the clear difference in the LFs for galaxies with different colors. The CNOC2 sample is an order of magnitude larger than any other redshift surveys in this redshift range (e.g., CFRS: Lilly et al. 1995; Autofib: Ellis et al. 1996). This large sample size, in combination with extensive $U B g R I$ photometry (plus $K$ for some fields), will permit precise measurements of the LF and its evolution with redshift for different galaxy populations.

Currently, various projects are being carried out using this large data base. We will be able to determine the galaxy correlation function in redshift and morphological bins, estimate the evolution of the pairwise velocity distribution, and measure the bias of galaxy clustering relative to mass clustering. The data are also being used to create a complete catalog of groups and pairs of galaxies, from which we can study the dynamics and evolution of galaxy groups and the redshift dependence of the merger rate. Line index measurements and principal component analysis of the spectra will be carried out, allowing us to conduct a detailed investigation of the star formation history of the galaxies. In addition, we will also derive quantitative morphological parameters of the galaxies, allowing us to examine the relationship between morphology, environment, and galaxy evolution. We will be able to obtain well-calibrated photometric redshifts for about 20,000 galaxies down to $R \sim 22.0$, which will further improve our studies of galaxy clustering and populations, and also extend the results to higher redshift.

\section{References}

Ellis, R.S., et al. 1996, MNRAS, 280, 235

2. Geller M. \& Huchra, J.P. 1989, Science, 246, 897

3. Lilly, S., Le Fèvre, O., Crampton, D., Hammer, F., \& Tresse, L. 1995, ApJ, 455, 50

4. Shectman S. et al. 1996, ApJ, 470, 172

5. Yee, H.K.C., Ellingson, E., Carlberg, R.G. 1996, ApJS, 102, 269 (YEC)

6. Yee, H.K.C. 1991, PASP, 103, 396 